\title{
Monocular Blindness in a Non-Ketotic Patient
}

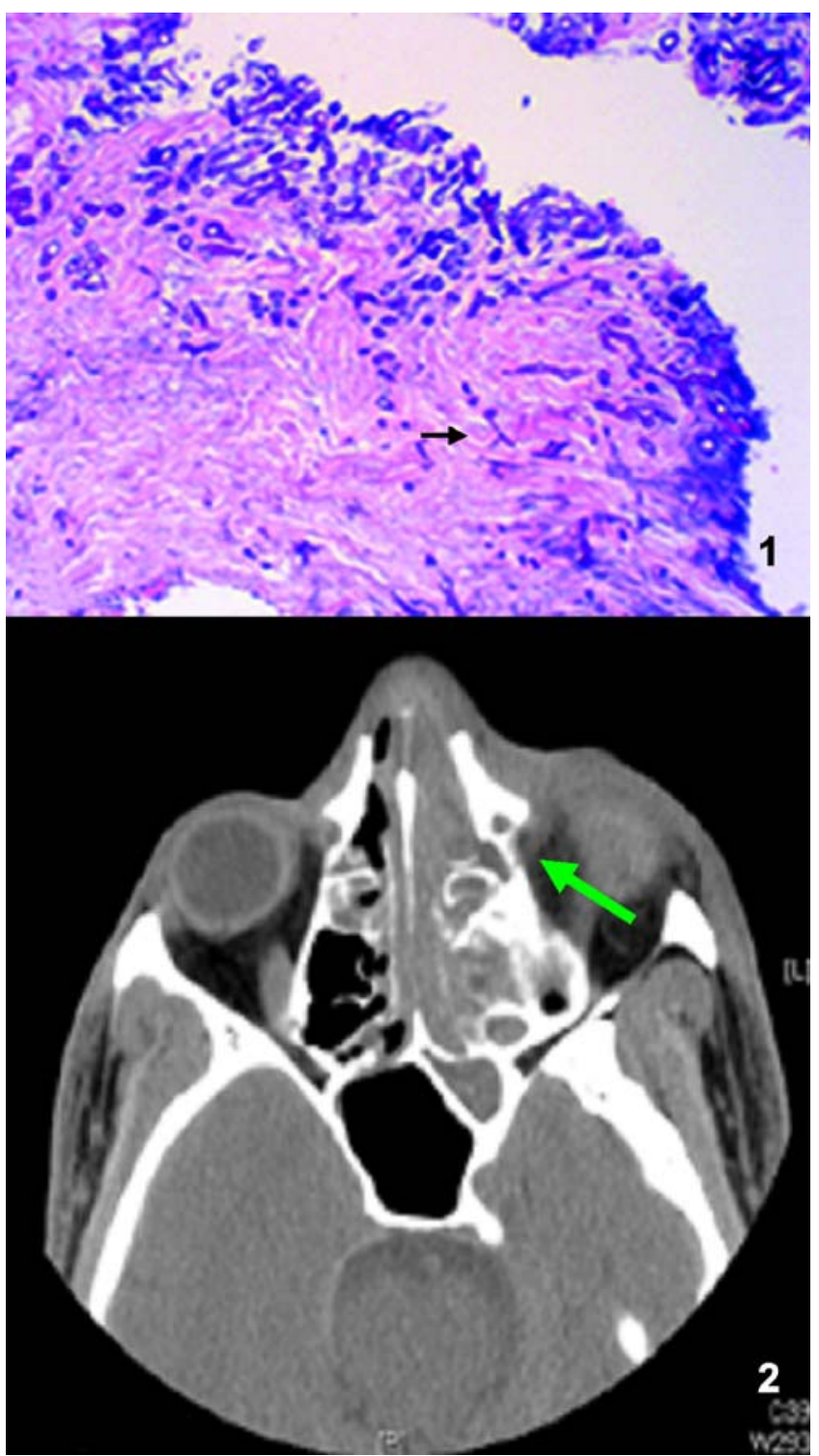

Figure 1. Open biopsy specimen showing a Mucor specie in $\mathrm{H} \& \mathrm{E}$ stain. Notice the hyphae, which are broad (5 to $15 \mathrm{~mm}$ diameter), irregularly branching at 90 degrees, and have rare septations.

Figure 2. CT scan of the orbits showing bilateral complete opacification of maxillary sinuses, left preseptal cellulitis, left periorbital cellulitis and subperiosteal phlegmon.

A 50 year-old male with a history of chronic renal insufficiency and diabetes mellitus type 2 presented with swelling, redness and pain of the left eye for two days.

He also started experiencing pain with the eye movement, itching and excessive lacrimation one day after the onset. Four hours prior to admission, he started having undocumented fever, bifrontal headaches, and blurred vision of the left eye. His mental status and vitals were unremarkable. He had proptosis, eyelid swelling, conjunctival erythema in the left eye associated with profuse serous discharge. Left eye vision was $20 / 200$. There was exquisite tenderness on the left orbit and periorbital region and extraocular muscle movements were painful. There was tenderness on both maxillary sinuses. Nasal mucosa was erythematous. Ear and throat examination were normal. Cardio-respiratory, gastrointestinal and neurological examinations were normal. Patient was started empirically on vancomycin, ceftazidime and metronidazole for orbital cellulitis. He was also started on intravenous dexamethasone for painful left eye blindness. Computerized tomography (CT) scan of the orbits revealed bilateral maxillary sinusitis (Figure 2) and preseptal and subperiosteal phlegmon involving the medial wall of the orbit and dehiscence of the left ethmoid sinus roof at the level of the cribriform plate. Bony erosion was noted in the superior aspect of the left medial wall of left orbit (Figure 2). MRI of the brain was normal. Initial laboratory values revealed uncontrolled diabetes mellitus with blood sugar of $415 \mathrm{mg} / \mathrm{dL}$ without evidence of diabetic ketoacidosis for which he was treated with insulin. White blood cell count was 19,400 cells/ $\mathrm{mm}^{3}$ with differential cell count of $85 \%$ neutrophils, $11 \%$ lymphocytes. Nasal and sinus endoscopy revealed posterior nasal septum necrosis and a possible abscess. Endoscopic nasal biopsy did not reveal any organisms. Over the course of the next few days he presented persistent fever. Urgent nasal and sinus endoscopy with biopsy and examination of maxillary drainage again did not reveal any organism. Open biopsy with anterior ethmoidectomy, left maxillary antrectomy, left total ethmoidectomy, left sphenoidectomy was performed, which showed nonseptate hyphae with right angle branching consistent with the diagnosis of mucormycosis (Figure 1). Extensive debridement surgery was performed, which revealed meningeal invasion. Patient was treated with systemic liposomal amphotericin B for three months. Patient survived extensive surgery but had significant disfigurement on a sixmonth follow-up.

Diagnosis of rhinocerebral mucormycosis should be considered in the clinical setting of monocular vision loss in a diabetic patient. Multiple biopsies or even open biopsy may be necessary if the clinical suspicion of mucormycosis is high. Early diagnosis and aggressive treatment leads to favorable outcome.

\footnotetext{
Archit Bhatt ${ }^{1}$, Bhavini Bhavsar ${ }^{2}$, Muhammad U. Farooq ${ }^{1}$ and Michelle Dahdouh ${ }^{3}$

${ }^{1}$ Michigan State University, Department of Neurology and Ophthalmology; ${ }^{2}$ Michigan State University, Department of Adult

Medicine; East Lansing, Michigan; ${ }^{3}$ St. Barnabas Hospital/Weill Medical College, Division of Infectious Diseases; Bronx, New York, USA
}

Received on 15 January 2008; revised 6 April 2008. Address for correspondence: Dr.Archit Bhatt. E-mail: archit.Bhatt@ht.msu.edu. The Brazilian Journal of Infectious Diseases 2008;12(2):161. (c) 2008 by The Brazilian Journal of Infectious Diseases and Contexto Publishing. All rights reserved. 